\title{
Symmetric bundles and representations of Lie triple systems
}

\author{
Wolfgang BERTRAM and Manon DIDRY \\ Institut Élie Cartan de Nancy, Université Nancy, CNRS, INRIA, \\ Boulevard des Aiguillettes, B.P. 239, F-54506 Vandœuvre-lès-Nancy, France \\ E-mails: bertram@iecn.u-nancy.fr, didrym@iecn.u-nancy.fr
}

\begin{abstract}
We define symmetric bundles as vector bundles in the category of symmetric spaces; it is shown that this notion is the geometric analog of the one of a representation of a Lie triple system. A symmetric bundle has an underlying reflection space, and we investigate the corresponding forgetful functor both from the point of view of differential geometry and from the point of view of representation theory. This functor is not injective, as is seen by constructing "unusual" symmetric bundle structures on the tangent bundles of certain symmetric spaces.
\end{abstract}

2000 MSC: 17A01, 17B10, 53C35.

\section{Introduction}

Although this is not common, linear representations of Lie groups may be defined as vector bundles in the category of Lie groups: if $\rho: G \rightarrow \mathrm{GL}(V)$ is a (say, finite-dimensional) representation of a Lie group in the usual sense, then the semidirect product $F:=G \ltimes V$ of $G$ and $V$ is a Lie group and at the same time a vector bundle over $G$ such that both structures are compatible in the following sense:

(R1) the projection $\pi: F \rightarrow G$ is a Lie group homomorphism,

(R2) the group law $F \times F \rightarrow F$ is a morphism of vector bundles, i.e., it preserves fibers and, fiberwise, group multiplication $F_{g} \oplus F_{h} \rightarrow F_{g h},(v, w) \mapsto v w$ is linear.

Conversely, given a vector bundle $F$ over $G$ with total space a Lie group and having such properties, the representation of $G$ can be recovered as the fiber $F_{e}$ over the unit element $e$ on which $G$ acts by conjugation. For instance, the tangent bundle $T G$ corresponds to the adjoint representation, and the cotangent bundle $T^{*} G$ to the coadjoint representation of $G$.

In this work we wish to promote the idea that this way of viewing representations is the good point of view when looking for a notion of "representation" for other categories of spaces which, like Lie groups, are defined by one or several "multiplication maps": somewhat simplified, a representation of a given object $M$ of such a category is a vector bundle over $M$ in the given category, where "vector bundle in the given category" essentially means that the analogs of (R1) and (R2) hold. In fact, this simple notion came out as a result of our attempts to find a "global" or "geometric" analog of the notion of representation of general n-ary algebraic structures: given a multilinear algebraic structure defined by identities, such as Lie, Jordan or other algebras or triple systems, Eilenberg [8] introduced a natural notion of (general) representation (which is widely used 
in Jordan theory, see $[12,17,18]) .{ }^{1}$ Essentially, a representation $V$ of such an $n$-ary algebra $\mathfrak{m}$ is equivalent to defining on the direct sum $V \oplus \mathfrak{m}$ an $n$-ary algebraic structure satisfying the same defining identities as $\mathfrak{m}$ and such that some natural properties hold, which turn out to be exactly the "infinitesimal analogs" of (R1) and (R2): for instance, $V$ will be an "abelian" ideal in $V \oplus \mathfrak{m}$, corresponding to the role of the fiber in a vector bundle. The archetypical example is given by the adjoint representation which is simply $\mathfrak{m} \oplus \varepsilon \mathfrak{m}$ with $\varepsilon^{2}=0$, the scalar extension of $\mathfrak{m}$ by dual numbers, which will of course correspond to the tangent bundle in the geometric picture. However, nothing guarantees in principle that there be a "coadjoint representation" and a "cotangent bundle in the given category"!

This approach is very general and has a wide range of possible applications: at least locally, any affine connection on a manifold gives rise to a smooth "multiplication map" (a local loop, see [20]), which by deriving gives rise to $n$-ary algebras, and hence may be "represented" by vector bundles. Concretely, we will show how all these ideas work for the most proeminent example of such structures, namely for symmetric spaces (here, the approach to symmetric spaces by Loos [16] turns out to be best suited; we recall some basic facts and the relation with homogeneous spaces $M=G / H$ in Chapter 2). Symmetric bundles are defined as vector bundles in the category of symmetric spaces (Section 2.3) and their infinitesimal analogs, representations of Lie triple systems are introduced (Chapter 3). These have already been studied from a purely algebraic point of view by Hodge and Parshall [11]. Another algebraic point of view (see [10]) features the aspect of representations of Lie algebras with involution (cf. Section 4.1), which we use to prove that, in the real finite-dimensional case, such representations are in one-to-one correspondence with symmetric bundles (Theorem 4.2). This result implies that the cotangent bundle $T^{*} M$ of a real finite-dimensional symmetric space is again a symmetric bundle - this is much less obvious than the corresponding fact for the tangent bundle, and it owes its validity to the fact that, on the level of representations of Lie triples systems, every representation admits a dual representation (Section 5.2). Whereas, among the algebraic constructions of new vector bundles from old ones, the dual and the direct sum constructions survive in the category of symmetric bundles, this is not the case for tensor products and hom-bundles: they have to replaced by other, more complicated, constructions (Chapter 5).

Compared to the case of group representations, a new feature of symmetric bundles is that they are "composed objects": for a Lie group, the group structure on $G \ltimes V$ and its structure of a homogeneous vector bundle are entirely equivalent. For symmetric bundles, the structure of a homogeneous vector bundle carries strictly less information than that of the symmetric bundle: let us assume $F$ is a symmetric bundle over a homogeneous symmetric space $M=G / H$; then $F$ carries two structures: it is a homogeneous symmetric space $F=L / K$, and, under the action of the smaller group $G$, it is a homogeneous vector bundle $G \times_{H} V$, with $V=F_{o}$ being the fiber over the base point $o=e H$. Basically, seeing $F$ as a homogeneous vector bundle only retains the representation of $H$ on $F_{o}$, whereas seeing $F$ as symmetric space $L / K$ takes into account the whole isotropy representation of the bigger group $K$. In other words, there is a forgetful functor from symmetric bundles to homogeneous vector bundles. Conversely, the following "extension problem" arises: given a homogeneous symmetric space $M=G / H$, which homogeneous vector

\footnotetext{
${ }^{1} \mathrm{~A}$ word of warning: in the literature, especially on Jordan algebras, there is some confusion in terminology; the notion of general representation differs very much from the idea of a representation to be a homomorphism "into some matrix realization". Unfortunately, the word "representation" is also used in this second sense for Jordan algebras (cf., e.g., [9]) and for symmetric spaces (see [3, I.5]); we suggest to replace this by the term "specialization", in the sense of "homomorphism into a special (i.e., matrix or operator) object".
} 
bundles (i.e., which H-representations) admit a compatible structure of a symmetric bundle? On the infinitesimal level of general representations of Lie triple systems, the forgetful functor appears as follows: a general representation of a Lie triple system $\mathfrak{m}$ consists of two trilinear maps $(r, m)$, and we simply forget the second component $m$ (Section 3.4). The extension problem is then: when does $r$ admit a compatible trilinear map $m$ such that $(r, m)$ is a representation of Lie triple systems? For a geometric interpretation of this problem, one notes that the trilinear map $r$ is of the type of a curvature tensor, and indeed one can prove that every symmetric bundle admits a canonical connection (Theorem 6.1) such that $r$ becomes its curvature tensor (Theorem 6.2). It seems thus that the representations of $H$ that admit an extension to a symmetric bundle are those that can themselves be interpreted as holonomy representation of a connection on a vector bundle. However, the situation is complicated by the fact that the canonical connection on $F$ does not determine completely the symmetric bundle structure on $F$.

We do not attack in this work the problem of classifying representations of, say, finite dimensional simple symmetric spaces; but we give a large class of examples of "unusual" symmetric bundle structures on tangent bundles (Chapter 7), thus showing that the above mentioned forgetful functor is not injective. In fact, as observed in [3], many (but not all) symmetric spaces $M=G / H$ admit, besides their "usual" complexification $M_{\mathbb{C}}=G_{\mathbb{C}} / H_{\mathbb{C}}$, another, "twisted" or "hermitian" one $M_{h \mathbb{C}}=L / K$. We show here that a similar construction works when one replaces "complexification" by "scalar extension by dual numbers" (replace the condition $i^{2}=-1$ by $\left.\varepsilon^{2}=0\right)$, and that in this way we obtain two different symmetric structures on the tangent bundle $T M$. A particularly pleasant example is the case of the general linear group $M=\operatorname{GL}(n, \mathbb{R})$ : in this case, the usual tangent bundle $T M$ is the group $\mathrm{GL}(n, \mathbb{R}[\epsilon])$ (scalar extension by dual numbers), whereas the "unusual" symmetric structure on the tangent bundle is obtained by realizing $T M$ as the homogeneous space $\operatorname{GL}(n, \mathbb{D}) / \mathrm{GL}(n, \mathbb{R}[\varepsilon])$, where $\mathbb{D}$ is some degenerate version of the quaternions (Theorem 7.1). We conjecture that, for real simple symmetric spaces, there are no other symmetric bundle structures on the tangent space than the ones just mentioned. In other words, we conjecture that the extension problem as formulated here is closely related to the "extension problem for the Jordan-Lie functor" from [3]; however, this remains a topic for future research.

The results presented in this paper partially extend results from the thesis [6], where a slightly different axiomatic definition of symmetric bundles was proposed in a purely algebraic setting, permitting to state the analog of Theorem 4.2 (equivalence of symmetric bundles and representations of Lie triple systems) in an algebraic framework (arbitrary dimension and arbitrary base field; see Theorem 2.1.2 in loc. cit.), based on results published in [7]. The present paper is independent from the results of [7], but nevertheless the framework still is quite general: our symmetric spaces are of arbitrary dimension and defined over very general topological base fields or rings $\mathbb{K}$ - for instance, the setting includes real or complex infinite dimensional (say, Banach) symmetric spaces or $p$-adic symmetric spaces (Section 2.1). We hope the reader will agree that, in the present case, this degree of generality does not complicate the theory, but rather simplifies it by forcing one to search for the very basic concepts.

After this work had been finished, we learned from Michael Kinyon that the question of defining "modules" for an object in a category had already been investigated by Beck in his thesis (see [2]; see also [1]): he defines a module to be an abelian group object in the slice category over the given object. It seems reasonable to conjecture that, in the cases considered here, this notion should agree with ours, but by lack of competence in category theory we have not been able to check this. 


\section{Symmetric bundles}

\subsection{Notation and general framework}

This work can be read on two different levels: the reader may take $\mathbb{K}=\mathbb{R}$ to be the real base field and understand by "manifold" finite-dimensional real manifolds in the usual sense; then our symmetric spaces and Lie groups are the same as in [16] or [13], or one may consider a commutative topological field or ring $\mathbb{K}$, having dense unit group $\mathbb{K}^{\times}$and such that 2 is invertible in $\mathbb{K}$; then we refer to [5] for the definition of manifolds and Lie groups over $\mathbb{K}$. Readers interested in the general case should just keep in mind that, in general,

- symmetric spaces need no longer be homogeneous (cf. Example 2.2 below),

- there is no exponential map and hence no general tool to "integrate" infinitesimal structures to local ones.

If we use such tools, it will be specifically mentioned that we are in the real (or complex) finite-dimensional case. In the sequel, the word linear space means "(topological) $\mathbb{K}$-module".

\subsection{Symmetric spaces and reflection spaces}

A reflection space ("Spiegelungsraum", introduced by Loos in [15]) is a smooth manifold $M$ together with a smooth "product map" $\mu: M \times M \rightarrow M,(x, y) \mapsto \mu(x, y)=: \sigma_{x}(y)$ satisfying, for all $x, y, z \in M$,

(S1) $\mu(x, x)=x$,

(S2) $\mu(x, \mu(x, y))=y$,

(S3) $\sigma_{x} \in \operatorname{Aut}(\mu)$, i.e. $\mu(x, \mu(y, z))=\mu(\mu(x, y), \mu(x, z))$.

The reflection space $(M, \mu)$ is called a symmetric space if in addition

(S4) for all $x \in M$, the differential $T_{x}\left(\sigma_{x}\right)$ of the "symmetry" $\sigma_{x}$ at $x$ is the negative of the identity of the tangent space $T_{x} M$.

In the real finite-dimensional case this is (via the implicit function theorem) equivalent to

(S4') for all $x \in M$, the fixed point $x$ of $\sigma_{x}$ is isolated.

Homomorphisms of such structures are smooth maps which commute with product maps. According to (S3), all maps of the form $\sigma_{x} \circ \sigma_{y}, x, y \in M$, are automorphisms; the subgroup $G(M)$ of $\operatorname{Aut}(M)$ generated by these elements is called the transvection group of $M$. Often one considers the category of reflection spaces, respectively, symmetric spaces with base point: a base point is just a distinguished point, often denoted by $x_{0}$ or $o$, and homomorphisms are then required to preserve base points. If $o \in M$ is a base point, one defines the quadratic map by

$$
Q:=Q_{o}: M \rightarrow \operatorname{Aut}(M), \quad x \mapsto Q(x):=\sigma_{x} \circ \sigma_{o}
$$

and the powers by $x^{2 k}:=Q(x)^{k}(o)$ and $x^{2 k+1}:=Q(x)^{k} x$.

Example 2.1. The group case. Every Lie group with the new multiplication $\mu(g, h)=g h^{-1} g$ is a symmetric space. 
Example 2.2. Homogeneous symmetric spaces. We say that a symmetric space is homogeneous if the group $G:=G(M)$ acts transitively on it and carries a Lie group structure such that this action is smooth. Let $o$ be a base point and $H$ its stabilizer, so that $M \cong G / H$. Then the map $\sigma: G \rightarrow G, g \mapsto \sigma_{o} \circ g \circ \sigma_{o}$ is an involution of $G$, and the multiplication map on $G / H$ is given by

$$
\sigma_{o}(g H)=\sigma(g) H, \quad \sigma_{g H}\left(g^{\prime} H\right)=g \sigma(g)^{-1} g^{\prime} H
$$

In finite dimension over $\mathbb{K}=\mathbb{R}$, every connected symmetric space is of this form, for a suitable involution $\sigma$ of a Lie group $G$ (see $[15,16])$.

Example 2.3. Linear symmetric spaces. Assume $V$ is a linear space over $\mathbb{K}$; we consider $V \times V$ as a linear space and thus write $V \oplus V$. Assume that $V$ carries a symmetric space structure $\mu: V \oplus V \rightarrow V$ which is a $\mathbb{K}$-linear map. Because of (S4), the symmetry $s_{0}=\mu(0, \cdot): V \rightarrow V$, being a linear map, must agree with its tangent map $-\mathrm{id}_{V}$. Then it follows that

$$
\begin{aligned}
\mu(v, w) & =\mu((v, v)-(0, v)+(0, w))=\mu(v, v)-\mu(0, v)+\mu(0, w) \\
& =v-(-v)-w=2 v-w
\end{aligned}
$$

Conversely, every linear space equipped with the multiplication map $\mu(v, w)=2 v-w$ is a symmetric space (group case $G=V$ ). With respect to the zero vector as base point, $Q(x)=\tau_{2 x}$ is translation by $2 x$, and the powers are $x^{k}=k x$.

Example 2.4. Polynomial symmetric spaces. In the same way as in the preceding example, we can consider linear spaces together with a symmetric structure which is a polynomial map $V \oplus V \rightarrow V$. See [6] for a theory of such spaces.

\subsection{Symmetric bundles}

A symmetric bundle (or, longer but more precise, symmetric vector bundle) is a vector bundle $\pi: F \rightarrow M$ such that

(SB1) $(F, \mu)$ and $\left(M, \mu_{M}\right)$ are symmetric spaces such that $\pi: F \rightarrow M$ is a homomorphism of symmetric spaces,

(SB2) for all $(p, q) \in M \times M$, the map induced by $\mu: F \times F \rightarrow F$ fiberwise,

$$
F_{q} \oplus F_{p} \longrightarrow F_{\mu(p, q)}, \quad(v, w) \longmapsto \mu(v, w)
$$

(which is well defined according to (SB1)), is linear.

Homomorphisms of symmetric bundles are vector bundle homomorphisms that are also homomorphisms of the symmetric spaces in question. Clearly, the concept of symmetric bundle could be adapted to other classes of bundles whenever the fibers belong to a category that admits direct products (e.g., multilinear bundles in the sense of [5]): it suffices to replace (SB2) by the requirement that the map $F_{q} \times F_{p} \rightarrow F_{\mu(p, q)}$ be a morphism in that category. Also, it is clear that such concepts exist for any category of manifolds equipped with binary, ternary or other "multiplication maps", such as generalized projective geometries (cf. [4]). However, in the sequel, we will stick to the case of vector bundles and symmetric spaces. 
A symmetric bundle is called trivial if it is trivial as a bundle, and if, as a symmetric space, it is simply the direct product of $M$ with a vector space. The first nontrivial example of a symmetric bundle is the tangent bundle $F:=T M$ of a symmetric space $(M, \mu)$ : as to (SB1), it is well known that $T M$ with product map $T \mu: T M \times T M \rightarrow T M$ is a symmetric space such that the canonical projection is a homomorphism and the fibers are flat subspaces (see [16] for the real finite-dimensional case and [5] for the general case). Property (SB2) follows immediately from the linearity of the tangent map $T_{(p, q)} \mu: T_{p} M \times T_{q} M \rightarrow T_{\mu(p, q)} M$.

\subsection{Some elementary properties of symmetric bundles}

For a symmetric bundle $F$ over $M$, the following holds:

(SB3) The symmetric space structure on the fiber $F_{x}=\pi^{-1}(x)$ over $x \in M$ coincides with the canonical symmetric space structure of the vector space $\left(F_{x},+\right)$ (i.e., $\mu(u, v)=2 u-v$ ).

(SB4) The zero-section $z: M \rightarrow F$ is a homomorphism of symmetric spaces. (Hence in the sequel we may identify $M$ with $z(M)$, and the use of the same letter $\mu$ for the multiplication maps of $M$ and $F$ does not lead to confusion.)

(SB5) For all $r \in \mathbb{K}$, the fiberwise dilation map

$$
(r)_{F}: F \rightarrow F, \quad v \mapsto r v
$$

is an endomorphism of the symmetric space $F$; for $r \in \mathbb{K}^{\times}$it is an automorphism.

In fact, for $p=q$, (SB2) says that the fiber $F_{p}$ is a symmetric subspace of $F$ such that its structure map $F_{p} \oplus F_{p} \rightarrow F_{p}$ is linear, and (SB3) now follows in view of Example 2.3. Since a linear map sends zero vector to zero vector, we have $\mu\left(0_{p}, 0_{q}\right)=0_{\mu(p, q)}$ proving (SB4), and to prove (SB5), just note that for $v \in F_{p}$ and $w \in F_{q}$, by (SB2),

$$
(r)_{F}(\mu(v, w))=r \mu(v, w)=\mu(r v, r w)=\mu\left((r)_{F} v,(r)_{F} w\right)
$$

In particular, note that $(0)_{F}$ is the projection onto the zero section, and that $(-1)_{F}$ can be seen as a "horizontal reflection with respect to the zero section".

\subsection{Horizontal and vertical symmetries}

Let $\pi: F \rightarrow M$ a symmetric bundle and $u \in F_{p}$. We define the horizontal (resp., vertical) symmetry (with respect to $u$ ) by

$$
\vartheta_{u}:=\sigma_{\frac{u}{2}} \circ(-1)_{F} \circ \sigma_{\frac{u}{2}}, \quad \nu_{u}:=\sigma_{\frac{u}{2}} \circ \sigma_{0_{p}} \circ(-1)_{F} \circ \sigma_{\frac{u}{2}}
$$

For $u=0_{p}$, the maps $\sigma_{u}, \vartheta_{u}$ commute with each other because of (SB5):

$$
(-1)_{F} \circ \sigma_{0_{p}} \circ(-1)_{F}=\sigma_{-0_{p}}=\sigma_{0_{p}}
$$

Therefore $\nu_{u}$ then is also is of order 2. Conjugating by $\sigma_{\frac{u}{2}}$, we see that for all $u \in F_{p}$, we get three pairwise commuting automorphisms $\sigma_{u}, \vartheta_{u}, \nu_{u}$, of order 2 and fixing the point $\sigma_{\frac{u}{2}}\left(0_{p}\right)=u$.

Lemma 2.5. The vertical symmetry depends only on the fiber $F_{p}$, that is, for all $u, w \in F_{p}$, we have $\nu_{u}=\nu_{w}=\nu_{0_{p}}=(-1)_{F} \circ \sigma_{0_{p}}$. 
Proof. Let us show that $\nu_{u}=\nu_{0}$ with $0=0_{p}$, i.e.,

$$
\sigma_{\frac{u}{2}} \circ(-1)_{F} \circ \sigma_{0} \circ \sigma_{\frac{u}{2}}=(-1)_{F} \circ \sigma_{0}
$$

But this follows from $\sigma_{0} \sigma_{\frac{u}{2}} \sigma_{0}=\sigma_{\sigma_{0}\left(\frac{u}{2}\right)}=\sigma_{-\frac{u}{2}}$ and the fact that $(-1)_{F}$ is an automorphism.

Proposition 2.6. The space $F$ together with the binary map $\nu: F \times F \rightarrow F,(v, w) \mapsto \nu_{v}(w)$ is a reflection space.

Proof. The defining properties (S1) and (S2) say that $\nu_{v}$ is of order 2 and fixes $v$, and this has already been proved above. In order to establish (S3), let $v \in F_{p}$ and $w \in F_{q}$. Then, using the preceding lemma,

$$
\begin{aligned}
\nu_{v} \nu_{w} \nu_{v} & =\nu_{0_{p}} \nu_{0_{q}} \nu_{0_{p}}=(-1)_{F} \circ \sigma_{0_{p}} \circ(-1)_{F} \circ \sigma_{0_{q}} \circ(-1)_{F} \circ \sigma_{0_{p}} \\
& =(-1)_{F} \circ \sigma_{0_{p}} \circ \sigma_{0_{q}} \circ \sigma_{0_{p}}=(-1)_{F} \circ \sigma_{\mu(p, q)}=\nu_{\nu_{v}(w)}
\end{aligned}
$$

We say that $(F, \nu)$ is the reflection space associated to the symmetric bundle $(F, \mu)$. Thus we get a functor $(F, \mu) \mapsto(F, \nu)$ from symmetric vector bundles to reflection spaces; it will be a recurrent theme in this work to interpret this functor as a forgetful functor. Note that the differential of $\nu_{u}$ has 1-eigenspace tangent to the fiber through $u$ and -1-eigenspace complementary to it; thus the distribution of the "vertical" 1-eigenspaces is integrable, whereas the distribution of the "horizontal" -1-eigenspaces is in general not (see Chapter 6: the curvature of the corresponding Ehresmann connection does in general not vanish).

\subsection{Automorphisms downstairs and upstairs}

The canonical projection $\pi: \operatorname{Aut}(F) \rightarrow \operatorname{Aut}(M),(\tilde{g}, g) \mapsto g$ does in general not admit a cross-section; we cannot even guarantee that it is surjective. However, it is easily seen that the projection of transvection groups $G(F) \mapsto G(M)$ is surjective: write $g \in G(M)$ as a composition of symmetries at points of $M$; identifying $M$ with the zero section in $F$ we see that $g$ gives rise to an element $\tilde{g} \in G(F)$ with $\pi(\tilde{g}, g)=g$. In particular, if $M$ is homogeneous, then so is $F$ : in fact, if $v \in F_{x}$, then there exists $g \in G(M)$ with $g . o=x$; then $\sigma_{\frac{v}{2}} \circ \tilde{g}\left(0_{o}\right)=v$. In the real finite-dimensional case, we may replace $G(M)$ by its universal covering; then the zero section $z: M \rightarrow F$ induces a homomorphism of this universal covering into $G(F)$, having discrete kernel. Hence, if we write $F=L / K$, it is not misleading to think of $G$ as a subgroup of $L$ and of $H$ as a subgroup of $K$ (possibly up to a discrete subgroup).

\subsection{Homogeneous bundles over symmetric spaces}

Assume that $M=G / H$ is a homogeneous symmetric space (Example 2.2). To any smooth action $H \times U \rightarrow U$ on a manifold $U$ one can associate the homogeneous bundle

$$
F=G \times{ }_{H} U=G \times U / \sim, \quad(g h, v) \sim(g, h v), \quad \forall h \in H
$$

When the base $M=G / H$ is a symmetric space, we define $\nu: F \times F \rightarrow F$ by

$$
\nu([f, v],[g, w])=\left[f \sigma(f)^{-1} \sigma(g), w\right]
$$

and one can show that $G \times_{H} U$ becomes a reflection space such that the projection onto $M$ becomes a homomorphism of reflection spaces (cf. [15, Theorem 1.5]). Let us say that then 
$F$ is a reflection space over the symmetric space $M$. The preceding formula shows that the reflection $\nu_{v}$ does not depend on the choice of $v \in U$, i.e., it depends only on the base. Loos has shown [15] that, conversely, every real finite-dimensional and connected reflection space can be written in this way as a homogeneous bundle over a symmetric space. In particular, linear representations of $H$ and reflection spaces over $M$ with linear fibers ("reflection vector bundles over $M ")$ correspond to each other.

Having this in mind, we now consider a symmetric bundle $\pi: F \rightarrow M$ over a homogeneous symmetric space $M=G / H$. As we have just seen, $F$ is then also homogeneous, say, $F=L / K$. Looking at $H$ as a subgroup of $K$ (see Section 2.6), we get a linear representation of $H$ on the fiber $F_{o}$, and we can write $F=G \times_{H} F_{o}$ as a homogeneous bundle over the base $M$. In this way, the functor from symmetric bundles to reflection spaces corresponds in the homogeneous case to the functor from symmetric bundles $F=L / K$ over $M=G / H$ to the associated homogeneous bundle $F=G \times_{H} F_{o}$. Conversely, we can formulate an extension problem: For which representations $H \rightarrow \mathrm{GL}(V)$ does the homogeneous bundle $F=G \times_{H} V$ carry a symmetric bundle structure? If it does, how many such structures are there?

\subsection{Derivations of symmetric bundles and vertical automorphisms}

A derivation of a symmetric bundle $F$ is a homomorphism of symmetric spaces $X: M \rightarrow F$ which at the same time is a smooth section of $\pi$ (see [16] for this terminology in case of the tangent bundle). A vertical automorphism of a symmetric bundle is an automorphism $f$ of the symmetric bundle $F$ preserving fibers, i.e., $\pi \circ f=\pi$. Clearly, $X:=f \circ z: M \rightarrow F$ then is a derivation of $F$. Conversely, if $X$ is a derivation, define

$$
f: F \rightarrow F, \quad F_{p} \ni v \longmapsto v+X(p)
$$

Then $f$ is a vertical automorphism: it is clearly smooth, preserves fibers, and is bijective. It is an automorphism: using (SB2),

$$
\begin{aligned}
\mu(f(v), f(w)) & =\mu(v+X(p), w+X(q))=\mu((v, w)+(X(p), X(q))) \\
& =\mu(v, w)+\mu(X(p), X(q))=\mu(v, w)+X \mu(p, q)=f(\mu(v, w))
\end{aligned}
$$

Summing up, vertical automorphisms are the same as derivations. Moreover, they clearly form a normal subgroup $\operatorname{Vaut}(F)$ in the group $\operatorname{Aut}(F)$, where composition corresponds to addition of sections. It follows that the space of derivations is stable under addition; it is also stable under multiplication by scalars, hence forms a vector group. The same kind of arguments shows that in fact we have an exact sequence

$$
0 \longrightarrow \operatorname{Vaut}(F) \longrightarrow \operatorname{Aut}(F) \longrightarrow \operatorname{Aut}(M) \longrightarrow 1
$$

(which essentially splits if we take transvection groups; cf. the discussion in Section 2.6). Now fix a base point $o \in M$; then the involution given by conjugation with $\sigma_{0_{o}}$ restricts to $\operatorname{Vaut}(F)$ and thus defines a linear map. Let us write

$$
\operatorname{Vaut}(F)=\operatorname{Vaut}^{+}(F) \oplus \operatorname{Vaut}^{-}(F)
$$

for the corresponding eigenspace decomposition. 
Lemma 2.7. We have $\operatorname{Vaut}^{+}(F)=\left\{f \in \operatorname{Vaut}(F) \mid f\left(0_{o}\right)=0_{o}\right\}$, and the map

$$
\operatorname{Vaut}^{-}(F) \longrightarrow F_{o}, \quad f \longmapsto f\left(0_{o}\right)
$$

is a bijection with inverse $v \mapsto \sigma_{\frac{v}{2}} \sigma_{0_{o}}$.

Proof. See [16] or [5, Proposition 5.9] for the proof in the case of the tangent bundle; the same arguments apply here.

\section{General representations of Lie triple systems}

Definition 3.1. A Lie triple system (Lts) is a linear space $\mathfrak{m}$ over $\mathbb{K}$ together with a trilinear map $\mathfrak{m} \times \mathfrak{m} \times \mathfrak{m} \rightarrow \mathfrak{m},(X, Y, Z) \mapsto[X, Y, Z]$ such that, writing also $R(X, Y)$ for the endomorphism $[X, Y, \cdot]$

(LT1) $R(X, Y)=-R(Y, X)$ (skew-symmetry),

(LT2) $R(X, Y) Z+R(Y, Z) X+R(Z, X) Y=0$ (the Jacobi identity),

(LT3) $R(X, Y)$ is a derivation of the trilinear product on $\mathfrak{m}$, i.e.,

$$
R(X, Y)[U, V, W]=[R(X, Y) U, V, W]+[U, R(X, Y) V, W]+[U, V, R(X, Y) W]
$$

For instance, if $(\mathfrak{g}, \sigma)$ is a Lie algebra with involution, then the -1-eigenspace $\mathfrak{m}$ of $\sigma$ with $[X, Y, Z]:=[[X, Y], Z]$ is an Lts. Every Lts arises in this way (see Section 4.1 below). For later use we introduce also the "middle multiplication operators" $M(X, Z) Y:=[X, Y, Z]$; then, in presence of (LT1), property (LT2) can be written in operator form

(LT2a) $M(X, Z)-M(Z, X)=R(X, Z)$

and similarly, reading (LT3) as an identity of operators, applied to the variable $W, V$ or $Y$, we get the following equivalent conditions, respectively:

$$
\begin{aligned}
& \text { (LT3a) }[R(X, Y), R(U, V)]=R(R(X, Y) U, V)+R(U, R(X, Y) V), \\
& \text { (LT3b) }[R(X, Y), M(U, W)]=M(R(X, Y) U, W)+M(U, R(X, Y) W), \\
& \text { (LT3c) } M(X, R(U, V) W)=-M(V, W) \circ M(X, U)+M(U, W) \circ M(X, V)+R(U, V) \circ M(X, W) .
\end{aligned}
$$

\subsection{The Lie triple system of a symmetric space}

Let $(M, \mu)$ be a symmetric space with base point $o$. Consider the tangent bundle $T M$ and write $\mathfrak{g}:=\operatorname{Vaut}(T M)$ for the derivations of the symmetric bundle $T M$, and $\mathfrak{g}=\mathfrak{h} \oplus \mathfrak{m}$ for the eigenspace decomposition from Lemma 2.7. One shows that $\mathfrak{g}$, seen as a space of vector fields on $M$, is stable under the Lie bracket and that $\sigma_{0}$ induces an involution of this Lie algebra structure. Hence the -1 -eigenspace $\mathfrak{m}$ is an Lts. Via the bijection $\mathfrak{m} \rightarrow T_{o} M, X \mapsto X(o)$ from Lemma 2.7 this Lts structure can be transferred to the tangent space $T_{o} M$, which, by definition, is the Lts associated to the pointed symmetric space $(M, o)$ (cf. [16] or [5, Chap 5]). The Lts depends functorially on $M$ and plays a similar role for symmetric spaces as the Lie algebra for a Lie group. (In particular, in the real finite-dimensional case there is an equivalence of categories between Lts and connected simply connected spaces with base point, cf. [16].) 


\subsection{The Lts of a symmetric bundle}

Now assume that $\pi: F \rightarrow M$ is a symmetric bundle over $M$ and fix a base point $o \in M$ and let $\mathfrak{f}:=T_{0_{o}} F$. Since $F$ is a symmetric space, $\mathfrak{f}$ is a Lie triple system. We wish to describe its structure in more detail. The differentials of the three involutions $\sigma_{0}, \vartheta_{0}$, and $\nu_{0}$ from Section 2.5 act by automorphisms on the Lts $f$ (where we write 0 instead of $0_{o}$ ). The +1 -eigenspace of $\vartheta$ can be identified with the Lts $\mathfrak{m}$, via the tangent map of the zero-section, and its -1-eigenspace is the tangent space $T_{0}\left(F_{o}\right)$ of the fiber $V=F_{o}$ which we identify with $V$ (in the notation from [5] we could also write $\varepsilon V$ for this "vertical space").

Lemma 3.2. The decomposition $\mathfrak{f}=\mathfrak{m} \oplus V$ has the following properties:

(1) $V$ is an ideal of $\mathfrak{f}$, i.e., $[x, y, z] \in V$ as soon as one of the $x, y, z$ belongs to $V$,

(2) $\mathfrak{m}$ is a sub-Lts of $\mathfrak{f}$,

(3) $[V, V, \mathfrak{m}]=[V, \mathfrak{m}, V]=0$,

(4) $V$ is abelian, i.e., $[V, V, V]=0$.

Proof. (1) is clear since $V$ is the kernel of a homomorphism (the differential $T_{0} \pi$ of $\pi$ at $0)$; (2) follows from the fact that $\mathfrak{m}$ is the fixed point space of the horizontal automorphism $T_{0}\left(\vartheta_{0}\right)=(-1)_{F}$ (moreover, we see that $\mathfrak{m}$ is isomorphically mapped by $T_{0} \pi$ onto the Lts of $M$ ), and (3) holds since $\tilde{\nu}:=T_{0}\left(\nu_{0}\right)$ is an automorphism and hence

$$
\tilde{\nu}[v, w, X]=[\tilde{\nu}(v), \tilde{\nu}(w), \tilde{\nu}(X)]=[v, w,-X]=-[v, w, X]
$$

hence $[v, w, X]$ belongs to $\mathfrak{m}$ and thus to $\mathfrak{m} \cap V=0$ since $V$ is an ideal. Finally, the fiber $F_{o}$ carries the "flat" symmetric space structure of a linear space and hence $[V, V, V]=0$.

A side-remark: if we adapt the whole set-up to the case of bilinear bundles (in the sense of [5]) instead of vector bundles, we get essentially the same properties, the only difference being that (3) does no longer hold: e.g., for the bilinear bundle TTM over $M$, the tangent model is $\mathfrak{m} \oplus\left(\varepsilon_{1} \mathfrak{m} \oplus \varepsilon_{2} \mathfrak{m} \oplus \varepsilon_{1} \varepsilon_{2} \mathfrak{m}\right)$, where the term in brackets is still an abelian Lts, but (3) does no longer hold: in fact, $\left[\varepsilon_{1} \mathfrak{m}, \varepsilon_{2} \mathfrak{m}, \mathfrak{m}\right]$ is nonzero in general.

\subsection{Representations and modules}

Let $\mathfrak{m}$ be an Lts. An $\mathfrak{m}$-module is a vector space $V$ such that the direct sum $\mathfrak{f}:=\mathfrak{m} \oplus V$ carries the structure of an Lts satisfying the properties from the preceding lemma. More explicitly, this means, by decomposing

$$
[X \oplus u, Y \oplus v, Z \oplus w]=[X, Y, Z] \oplus(r(X, Y) w+m(X, Z) v-m(Y, Z) u+[u, v, w])
$$

that we are given two trilinear maps $r$ and $m$ :

$$
\begin{array}{rlrl}
r: \mathfrak{m} \times \mathfrak{m} & \rightarrow \operatorname{End}(V), & r(X, Y)=[X, Y, \cdot] \\
m: \mathfrak{m} \times \mathfrak{m} \rightarrow \operatorname{End}(V), & m(X, Z)=[X, \cdot, Y]=-[\cdot, X, Y]
\end{array}
$$

satisfying the properties given by the following lemma.

Lemma 3.3. For any Lts $(\mathfrak{m}, R)$, the space $\mathfrak{m} \oplus V$ with a triple bracket given by (3.1) is an Lts if and only if $r$ and $m$ satisfy the following relations: 


$$
\begin{aligned}
\text { (R1) } & r(X, Y)=-r(Y, X), \\
\text { (R2) } & m(X, Z)-m(Z, X)=r(X, Z), \\
\text { (R3) } & r(R(X, Y) U \otimes V+U \otimes R(X, Y) V)=[r(X, Y), r(U, V)] \\
& m(R(X, Y) U \otimes V+U \otimes R(X, Y) V)=[r(X, Y), m(U, V)], \\
\text { (R4) } & m(X, R(U, V) W)-r(U, V) \circ m(X, W)=m(U, W) \circ m(X, V)-m(V, W) \circ m(X, U) .
\end{aligned}
$$

Proof. We have to show that (LT1)-(LT3) for $\mathfrak{m} \oplus V$ are equivalent to (R1)-(R4): first of all, we note that a bracket is zero if more than one of the three arguments belongs to $V$. Now, (LT1) is equivalent to (R1) if both arguments belong to $\mathfrak{m}$ and holds by (3.1) if one is in $V$ and the other in $\mathfrak{m}$. Next, (LT2) is an identity in three variables. We may assume that two variables, say $X$ and $Z$, belong to $\mathfrak{m}$, and write (LT2) in its operator form (LT2a). Thus we see that (LT2) is equivalent to (R2). Finally, (LT3) is an identity in 5 variables. In order to get a nontrivial identity, we can assume that at least four of them belong to $\mathfrak{m}$. We then write (LT3) in operator form (identities (LT3a,b,c) from Definition 3.1), and see that (LT3) is equivalent to (R3) and (R4), thus proving our claim.

Note that, in view of (R3), identity (R4) is equivalent to the following identity:

(R4') $m(R(U, V) X, W)-m(X, W) \circ r(U, V)=m(V, W) \circ m(X, U)-m(U, W) \circ m(X, V)$.

Condition (R4) can be rephrased by saying that the operator $R(X, v)$ defined by $R(X, v) Y=$ $m(X, Y) v$ belongs to the space of derivations from $\mathfrak{m}$ into $V$,

$$
\begin{aligned}
\operatorname{Der}(\mathfrak{m}, V)=\{ & D: \mathfrak{m} \longrightarrow V \mid \forall X, Y, Z \in \mathfrak{m}: \\
& D R(X, Y, Z)=r(X, Y) D Z+m(X, Z) D Y-m(Y, D X) Z\}
\end{aligned}
$$

Definition 3.4. A general representation of a Lie triple system $\mathfrak{m}$ in a unital associative algebra $A$ is given by two bilinear maps

$$
\begin{aligned}
r: \mathfrak{m} \times \mathfrak{m} & \longrightarrow A, \quad(X, Y) \longmapsto r(X, Y) \\
m: \mathfrak{m} \times \mathfrak{m} \longrightarrow A, \quad(X, Z) & \longmapsto m(X, Z)
\end{aligned}
$$

such that (R1)-(R4) hold (where $\circ$ has to be interpreted as the product in $A$ and the bracket is the Lie bracket in $A$ ). If $A=\operatorname{End}(V)$ is the endomorphism algebra of a vector space, we say that $V$ is an $\mathfrak{m}$-module. Homomorphisms of $\mathfrak{m}$-modules are defined in the obvious way, thus turning $\mathfrak{m}$-modules into a category. Given an $\mathfrak{m}$-module $V$, the Lts $\tilde{\mathfrak{m}}=\mathfrak{m} \oplus V$ with bracket defined by (3.1) is called the split null extension of $\mathfrak{m}$ by the module $V$. It is fairly obvious that the split null extension depends functorially on the m-module $V$.

Example 3.5 (regular representation). For any Lts $\mathfrak{m}$, consider its "extension by dual numbers", i.e., let $\mathbb{K}[\varepsilon]=\mathbb{K}[X] /\left(X^{2}\right)=\mathbb{K} \oplus \varepsilon \mathbb{K}, \varepsilon^{2}=0$ (ring of dual numbers over $\mathbb{K}$ ), and

$$
\tilde{\mathfrak{m}}=\mathfrak{m} \otimes_{\mathbb{K}} \mathbb{K}[\varepsilon]=\mathfrak{m} \oplus \epsilon \mathfrak{m}
$$

with the $\epsilon$-trilinear extension of the bracket from $\mathfrak{m}$ :

$$
\left[X+\epsilon X^{\prime}, Y+\epsilon Y^{\prime}, Z+\epsilon Z^{\prime}\right]=[X, Y, Z]+\epsilon\left(\left[X, Y, Z^{\prime}\right]+\left[X, Y^{\prime}, Z\right]+\left[X^{\prime}, Y, Z\right]\right)
$$


This is nothing but the split null extension of $\mathfrak{m}$ by the regular representation, which by definition is given by $V=\mathfrak{m}$ and

$$
r(X, Y)=R(X, Y): \mathfrak{m} \longrightarrow \mathfrak{m}, \quad m(X, Y)=M(X, Y): \mathfrak{m} \longrightarrow \mathfrak{m}
$$

If $M$ is a symmetric space, then the Lts of the tangent bundle $T M$ is precisely $\mathfrak{m} \oplus \varepsilon \mathfrak{m}$ (cf. [5]). Hence the regular representation corresponds to the tangent bundle of $M$.

\subsection{The extension problem revisited}

The forgetful functor associating to a symmetric vector bundle its underlying reflection space corresponds to the forgetful functor $(r, m) \mapsto r$. Namely, if $\mathfrak{h}$ is the image of the skew-symmetric map

$$
\mathfrak{m} \otimes \mathfrak{m} \longrightarrow \operatorname{End}(\mathfrak{m}), \quad X \otimes Y \longmapsto R(X, Y)
$$

then (LT3) implies that $\mathfrak{h}$ is a Lie algebra, and for any representation $\rho: \mathfrak{h} \rightarrow \mathfrak{g l}(V)$, we may define $r(X, Y):=\rho(R(X, Y))$; then the first relation of (R3) is equivalent to $\rho$ being a representation. Thus we get the infinitesimal version of a homogeneous vector bundle. Now the problem of finding a compatible symmetric vector bundle structure corresponds to finding the second component $m$ such that $(r, m)$ defines a representation of $\mathfrak{m}$.

\section{Reconstruction}

We have shown that a representation of an Lts is the derived version of a symmetric bundle. Conversely, can one reconstruct a symmetric bundle from a representation of an Lts? As a first step, it is always possible to recover Lie algebras from Lie triple systems, and certain Lie algebra representations from Lie triple representations. The second step is then to lift these constructions to the space level: here we have to make assumptions on the base field and on the topological nature of $M$.

\subsection{From Lie triple systems to Lie algebras with involution}

Lie triple systems and $\mathbb{Z} / 2 \mathbb{Z}$-graded Lie algebras: the standard imbedding. Every Lie algebra $\mathfrak{g}$ together with an involution $\sigma$ gives rise to an Lts $\mathfrak{m}=\mathfrak{g}^{-\sigma}$, equipped with the triple Lie bracket $[[X, Y], Z]$. Conversely, every Lts $\mathfrak{m}$ can be obtained in this way: let $\mathfrak{h}$ be the subalgebra of the algebra of derivations of $\mathfrak{m}$ generated by the endomorphisms $R(x, y), x, y \in \mathfrak{m}$. Then the space $\mathfrak{g}:=\mathfrak{h} \oplus \mathfrak{m}$ carries a Lie bracket given by

$$
\left[(D, X),\left(D^{\prime}, X^{\prime}\right)\right]:=\left(\left[D, D^{\prime}\right]+R\left(X, X^{\prime}\right), D X^{\prime}-D^{\prime} X\right)
$$

This Lie algebra, called the standard imbedding of the Lts $\mathfrak{m}$, does in general not depend functorially on $\mathfrak{m}$; see $[11,21]$ for a detailed study of functorial properties related to this and other constructions. Note that, in terms of the Lie algebra $\mathfrak{g}$, we can write

$$
R(X, Y)=\left.\operatorname{ad}[X, Y]\right|_{\mathfrak{m}}, \quad M(X, Z)=\left.\operatorname{ad}(Z) \circ \operatorname{ad}(X)\right|_{\mathfrak{m}}
$$


$\mathfrak{g}$-modules with involution. Assume $(\mathfrak{g}, \sigma)$ is a Lie algebra with involution. A representation $\rho: \mathfrak{g} \rightarrow \mathfrak{g l}(W)$ is called a $(\mathfrak{g}, \sigma)$-module with involution if $W$ is equipped with a direct sum decomposition $W=W^{+} \oplus W^{-}$which is compatible with $\sigma$ in the sense that

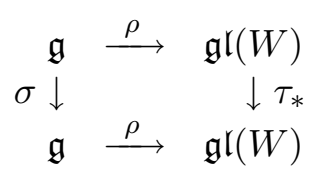

commutes, where $\tau \in \mathrm{GL}(W)$ is the identity on $W^{+}$and -1 on $W^{-}$, and $\tau_{*}(X)=\tau X \tau$.

Lemma 4.1. Let $\mathfrak{m}$ be an Lts and $\mathfrak{g}$ its standard imbedding. There exists a bijection between $(\mathfrak{g}, \sigma)$-modules with involution and $\mathfrak{m}$-modules.

Proof. Given a $(\mathfrak{g}, \sigma)$-module with involution $(W, \tau)$, we first form the semidirect product $\mathfrak{b}:=$ $\mathfrak{g} \ltimes W$. This is a Lie algebra carrying an involution given by $\sigma \times \tau$. Its -1-eigenspace $\mathfrak{m} \oplus W^{-}$is an Lts satisfying the relations from Lemma 3.2, and hence is the split null extension corresponding to an $\mathfrak{m}$-module $W^{-}$.

Given an $\mathfrak{m}$-module $V$, we construct first the split null extension $\mathfrak{m} \oplus V$ and then its standard imbedding $\mathfrak{b}=(\mathfrak{m} \oplus V) \oplus[\mathfrak{m} \oplus V, \mathfrak{m} \oplus V]$. Then

$$
W:=W^{+} \oplus W^{-}:=[V, \mathfrak{m}] \oplus V
$$

is a $\mathfrak{g}$-module with involution.

Again, the correspondence set up by the lemma is functorial in one direction but not in the other; see [11] for this issue.

\subsection{From modules to bundles}

Theorem 4.2. Let $\mathbb{K}=\mathbb{R}$ and $M$ be a finite-dimensional connected simply connected symmetric space with base point $o$. Let $\mathfrak{m}$ be its associated Lts and $\mathfrak{g}$ its standard imbedding, with involution $\sigma$. Then the following objects are in one-to-one correspondence:

(1) (finite-dimensional) symmetric vector bundles over $M$,

(2) (finite-dimensional) ( $\mathfrak{g}, \sigma)$-modules with involution,

(3) (finite-dimensional) $\mathfrak{m}$-modules.

The bijection between (1) and (3) is an equivalence of categories.

Proof. We have already seen how to go from (1) to (3), and that (2) and (3) are in bijection. Let us give a construction from (2) and (3) to (1): let $\mathfrak{f}=\mathfrak{m} \oplus V$ be the split null extension coming from an $\mathfrak{m}$-module $V$ and $\mathfrak{b}=\mathfrak{f} \oplus[\mathfrak{f}, \mathfrak{f}]$ the standard imbedding of $\mathfrak{f}$ and let $W$ be the corresponding $\mathfrak{g}$-module with involution. Let $G$ be the simply connected covering of the transvection group $G(M)$ and write $M=G / H$. Then the representation of $\mathfrak{g}$ on $W$ integrates to a representation of $G$ on $W$. Let

$$
B:=G \ltimes W, \quad K:=H \ltimes W^{+}, \quad F:=B / K
$$


We claim that

(i) $F$ is a vector bundle over $M$, isomorphic to the homogeneous bundle $G \times{ }_{H} W^{-}$,

(ii) $F$ carries the structure of a symmetric bundle over $M$.

Proof of (i): first of all,

$$
G W / H W^{+} \longrightarrow G \times_{H}\left(W / W^{+}\right), \quad g w / H W^{+} \longmapsto\left[\left(g, w / W^{+}\right)\right]
$$

is a well-defined bijection. Since $W / W^{+}=W^{-}$, this proves the first claim.

Proof of (ii): the Lie algebra $\mathfrak{k}$ of $K$ is the fixed point space of an involution of $\mathfrak{b}$, and hence $F=B / K$ is a symmetric space. Its Lts is $\mathfrak{f}$. The projection map $F \rightarrow M$ has as differential the projection from $\mathfrak{f}$ to $\mathfrak{m}$ and hence is a homomorphism of symmetric spaces.

Let us show that the structure map $F_{p} \oplus F_{q} \rightarrow F_{\mu(p, q)}$ is linear. Since we already know that $F$ is a homogeneous $G$-bundle, we may assume that $p=o$ is the base point. Now we proceed in two steps:

(a) we show that $\sigma_{0_{o}}: F_{\sigma(q)} \rightarrow F_{q}$ is linear. In fact, here we use that $W$ is a $G$-module with involution, i.e., $\sigma(g) w=\tau g \tau(w)$ :

$$
\begin{aligned}
\sigma([(g, w)]) & =\sigma(g w) / H W^{+}=\sigma(g) \sigma(w) / H W^{+} \\
& =\sigma(g)(-w) / H W^{+}=\tau(g(w)) / H W^{+}=-g(w) / H W^{+}=[(g,-w)]
\end{aligned}
$$

Thus this map is described by $w \mapsto-w$ and thus is linear.

(b) Since $\sigma_{0_{o}}: F_{\sigma(q)} \rightarrow F_{q}$ is a linear bijection, $F_{o} \oplus F_{q} \rightarrow F_{\sigma(q)}$ is linear (and well defined) if and only if so is the map $F_{o} \oplus F_{q} \rightarrow F_{q},(u, w) \mapsto \sigma_{0_{o}} \sigma_{u}(w)$. But the last map is the same as $(u, w) \mapsto(-2 u) . w$ (the point stands for the action of $W^{-}$on $F$; recall that in every symmetric space $\left.\sigma_{o} \sigma_{g . o}=\sigma(g) g^{-1}\right)$. Summing up, it suffices now to show that the map

$$
W^{-} \times F_{q} \longrightarrow F_{q}, \quad(u, z) \longmapsto u \cdot z
$$

is well defined and linear. Proof of this: let $u \in W^{-}$and $q=g H,[(g, w)] \in F_{q}$ with $w \in W^{-}=$ $W / W^{+}$. Then

$$
\begin{aligned}
u \cdot[(g, w)] & =u \cdot g w / H W^{+}=g g^{-1} u g w / H W^{+}=g\left(\rho(g)^{-1} u\right) w / H W^{+} \\
& =\left[\left(g, w+\operatorname{pr}_{-}\left(\rho(g)^{-1} u\right)\right)\right]
\end{aligned}
$$

Thus our map is described by $(u, w) \mapsto w+\alpha(u)$ with a linear map $\alpha \in \operatorname{End}\left(W^{-}\right)$that depends on $g$, and hence is linear, proving claim (ii).

Finally, the fact that homomorphisms in the categories defined by (1) and (3) correspond to each other follows from the corresponding fact for (connected simply connected) symmetric spaces and Lie triple systems, see [16].

\section{$5 \quad$ Linear algebra and representations}

So far we do not know any representations other than the regular one and the trivial ones. In the following we discuss the standard linear algebra constructions producing new representations from old ones. 


\subsection{Direct sums}

Clearly, if $\left(V, r_{1}, m_{1}\right)$ and $\left(W, r_{2}, m_{2}\right)$ are $\mathfrak{m}$-modules, then $\left(V \oplus W, r_{1} \oplus r_{2}, m_{1} \oplus m_{2}\right)$ is again a general representation. Correspondingly, the direct sum of symmetric bundles can be turned into a symmetric bundle.

\subsection{The dual representation}

If $(V, r, m)$ is an $\mathfrak{m}$-module, then the dual space $V^{*}$ can be turned into an $\mathfrak{m}$-module by putting

$$
r^{*}(X, Y):=-r(X, Y)^{*}=r(Y, X)^{*}, \quad m^{*}(X, Y):=m(Y, X)^{*}
$$

where $A^{*}: V^{*} \rightarrow V^{*}, \phi \mapsto \phi \circ A$ is the dual operator of an operator $A \in \operatorname{End}(V)$. In fact, the properties (R1)-(R3) for $\left(V^{*}, r^{*}, m^{*}\right)$ are easily verified; for (R4) note that (R4') written out for the dual is precisely (R4).

Equivalently: if $(\rho, \mathfrak{g}, V, \tau)$ is a $(\mathfrak{g}, \sigma)$-module with involution, one verifies that the dual module, $\rho^{*}(X)=-\rho(X)^{*}$, also is a module with involution $\tau^{*}$. It follows that

$$
m^{*}(X, Y)=\rho^{*}(X) \rho^{*}(Y)=\left(-\rho(X)^{*}\right)\left(-\rho(Y)^{*}\right)=(\rho(Y) \rho(X))^{*}=m(Y, X)^{*}
$$

leading to Formula (5.1).

In particular, in the finite-dimensional real case, invoking Theorem 3.4, the dual of the regular representation corresponds to the cotangent bundle $T^{*} M$ which thus again carries a symmetric bundle structure. (If $M=G / H$, then we may also write $T M=T^{*} G / T^{*} H$ where $T^{*} G=G \ltimes \mathfrak{g}^{*}$.) It remains intriguing that there seems to be no really intrinsic construction of this symmetric space structure on $T^{*} M$. For this reason we cannot affirm that (in cases where a reasonable topological dual $\mathfrak{m}^{*}$ of $\mathfrak{m}$ exists), in the infinite-dimensional case or over other base fields than $\mathbb{R}$ or $\mathbb{C}, T^{*} M$ is again a symmetric space.

\subsection{A duality principle}

Note that, for finite-dimensional modules over a field, $V$ is the dual of its dual module $V^{*}$. More generally, we can define for any general representation of $\mathfrak{q}$ in an algebra $A$ its opposite representation in the algebra $A^{\text {opp }}$ by putting

$$
r^{o p p}(X, Y)=r(Y, X), \quad m^{o p p}(X, Y)=m(Y, X)
$$

as above it is seen that this is again a representation. As an application of these remarks we get a duality principle similar as the one for Jordan pairs formulated by Loos (cf. [18]):

Proposition 5.1. If $I$ is an identity in $R(X, Y)$ and $M(U, V)$ valid for all Lie triples over $\mathbb{R}$, then its dual identity $I^{*}$, obtained by replacing $R(X, Y)$ by $R(Y, X)$ and $M(U, V)$ by $M(V, U)$ and reversing the order of all factors, is also valid for all Lie triples over $\mathbb{R}$.

Proof. If $I$ is valid for all Lts, then it is also valid for all split null extensions obtained from representations and hence the corresponding identity, with $R(X, Y)$ replaced by $r(X, Y)$ and $M(X, Y)$ by $m(X, Y)$, is valid for all representations. Since the set of all representations is the same as the set of all opposite representations, and since the opposite functor changes order of factors and order of arguments, we see that $I^{*}$ is valid for all representations. In particular, it is valid for the regular representation and hence holds in $\mathfrak{q}$. 
For instance, identities (R4) and (R4') (cf. Lemma 3.3 and remark following it) are dual in the sense of the proposition. We don't know about any application of Proposition 5.1; however, one may note that the original definition of Lie triple systems by Jacobson [12] as well as the exposition by Lister [14] are based on a set of five identities, among which two identities are equivalent to each other by the duality principle; they correspond to (LT3c) and its dual identity.

\subsection{Tensor products}

The tensor product $F_{1} \otimes F_{2}$ of two symmetric vector bundles is in general no longer a symmetric vector bundle: let $A:=\left(F_{1}\right)_{o}, B=\left(F_{2}\right)_{o}$ be the two fibers in question, regarded as $\mathfrak{m}$-modules. Extend $A$ and $B$ to $(\mathfrak{g}, \sigma)$-modules with involution, $V=V_{+} \oplus V_{-}, A=V_{-}, V_{+}=\operatorname{Der}(\mathfrak{m}, A)$, $W=W_{+} \oplus W_{-}, B=W_{-}, W_{+}=\operatorname{Der}(\mathfrak{m}, B)$. It is easily verified that then $\left(V \otimes W, \tau_{V} \otimes \tau_{W}\right)$ is again a $(\mathfrak{g}, \sigma)$-module with involution. Now, the minus-part in

$$
V \otimes W=\left(V_{+} \otimes W_{+} \oplus V_{-} \otimes W_{-}\right) \oplus\left(V_{+} \otimes W_{-} \oplus V_{-} \otimes W_{+}\right)
$$

is

$$
A \odot B:=A \otimes \operatorname{Der}(\mathfrak{m}, B) \oplus B \otimes \operatorname{Der}(\mathfrak{m}, A)
$$

which therefore is another m-module, replacing the ordinary tensor product $A \otimes B$. The corresponding definition of the maps $r$ is

$$
r(X, Y)(a \otimes D)=r_{A}(X, Y) a \otimes D+a \otimes\left(r_{B}(X, Y) \circ D-D \circ R(X, Y)\right)
$$

and there is a similar expression for the $m$-components and for $r(X, Y)\left(b \otimes D^{\prime}\right), m(X, Y)\left(b \otimes D^{\prime}\right)$. It is obvious that the operation $\odot$ is compatible with direct sums, and it also associative (in the same sense as the usual tensor product): the minus-part both in $U \otimes(V \otimes W)$ and in $(U \otimes V) \otimes W$ is

$$
U_{-} \odot V_{-} \odot W_{-}=\bigoplus_{i j k=-1} U_{i} \otimes V_{i} \otimes W_{i}
$$

where $i, j, k \in\{ \pm 1\}$ and with $U_{+}=\operatorname{Der}\left(\mathfrak{q}, U_{-}\right)$, etc.

\subsection{Hom-bundles}

If $A$ and $B$ are $\mathfrak{m}$-modules, then $\operatorname{Hom}(A, B)$ is in general not an $\mathfrak{m}$-module, but we can use the same construction as in Section 5.4 to see that

$$
\operatorname{Hom}(A, \operatorname{Der}(\mathfrak{m}, B)) \oplus \operatorname{Hom}(B, \operatorname{Der}(\mathfrak{m}, A))
$$

is again an $\mathfrak{m}$-module.

\subsection{Universal bundles}

Various definitions of universal or enveloping algebras (resp., universal representations), attached to triple systems or algebras with involution, can be given; see [10, 17, 18, 19]. These objects should correspond to certain "universal bundles" over a given symmetric space $M$. We intend to investigate such questions elsewhere. 


\section{The canonical connection of a symmetric bundle}

We now study in more detail the differential geometric aspects of symmetric bundles. It is immediately clear from Section 2.5 that a symmetric bundle $F$ carries a fiber bundle connection in the general sense of Ehresmann, i.e., there is a distribution of horizontal subspaces, complementary to the vertical subspaces $V_{u}=T_{u}\left(F_{p}\right)$ (tangent spaces of the fiber); namely, as horizontal subspace take the fixed point spaces of the differentials of the horizontal symmetry $\vartheta_{u}$,

$$
H_{u}=\left\{v \in T_{u} F \mid \vartheta_{u}(v)=v\right\}=Q\left(\frac{u}{2}\right)\left(T_{p} M\right)=\sigma_{\frac{u}{2}}\left(T_{p} M\right)
$$

In the sequel, we show that this Ehresmann connection is indeed a linear connection (in the general sense defined in [5], which in the real case amounts to the usual definitions), and that in general it has nonvanishing curvature, so that the distribution $\left(H_{u}\right)_{u \in F}$ is in general not integrable.

Theorem 6.1. Let $\pi: F \rightarrow M$ be a symmetric bundle over $M$. Then there exists a unique linear connection on the vector bundle $F$ which is invariant under all symmetries $\sigma_{x}, x \in M$.

Proof. The proof is similar to the one of [5, Theorem 26.3], and therefore will not be spelled out here in full detail. The uniqueness statement is proved by observing that the difference $A=L_{1}-L_{2}$ of two linear connections on $F$ is a tensor field such that $A_{p}: T_{p} M \times F_{p} \rightarrow F_{p}$ is bilinear; if both $L_{1}$ and $L_{2}$ are invariant under $\sigma_{p}$, then $A_{p}(-v,-w)=-A_{p}(w)$ and hence $A_{p}=0$. It follows that $L_{1}=L_{2}$. The main argument for the proof of existence consists in proving that the fibers of the bundle $T F$ over $M$ are abelian symmetric spaces (for this one has to analyze the map $T \mu: T F \times T F \rightarrow T F$ in the fiber over a point $p \in M$ in the same way as the corresponding map $(T T M \times T T M)_{p} \rightarrow(T T M)_{p}$ was analyzed in [5, Lemma 26.4]); then one concludes by general arguments that the fibers of $T F$ over $M$ carry canonically the structure of a linear space which is bilinearly related to all linear structures induced by bundle charts. By definition, this is what we call a linear connection on $F$.

\subsection{Extension problem: on uniqueness}

When $F=T M$ is the tangent bundle, with its canonical symmetric bundle structure, the connection defined by the preceding theorem is precisely the canonical connection of the symmetric space $M$ (cf. $[5,16])$. We will see in the next chapter that the abstract bundle $T M$ may carry several different (nonequivalent) symmetric bundle structures over $M$. The uniqueness statement of the theorem shows that they all lead to the same linear connection on $T M$ over $M$. Therefore the symmetric bundle structure is not uniquely determined by the linear connection from Theorem 6.1. Only at second order, by considering connections on TTM over $T M$, one is able to distinguish two symmetric bundle structures on $T M$.

Theorem 6.2. Let $\Omega$ be the curvature tensor of the linear connection on the symmetric bundle $F$ over $M$ defined in the preceding theorem. Then $\Omega$ is given by the $r$-component of the corresponding Lts representation: for all sections $X, Y$ of $T M$ and sections $\zeta$ of $F$,

$$
\Omega(X, Y) \zeta=r(X, Y) \zeta
$$

(i.e., with respect to an arbitrary base point $o \in M$, for all $v, w \in T_{o} M$ and $z \in F_{o}, \Omega_{o}(v, w) z=$ $r(v, w) z)$. 
Proof. In case $F=T M$, where $\Omega$ is the curvature of the canonical connection of $M$, it is well known that $\Omega$ is (possibly up to a sign, which is a matter of convention) given by the Lie triple system of $M$, i.e., $\Omega(X, Y) Z=R(X, Y) Z=[X, Y, Z]$, see $[16,13,3,5]$ for three different proofs. It is inevitable to go into third-order calculations, and therefore none of these proofs is really short. Theorem 6.2 generalizes this result, and it can also be proved in several different ways. We will here just present the basic ideas and refer the reader to the above references for details.

(a) Approaches using sections. Recall that the curvature $\Omega$ may be defined by

$$
\Omega(X, Y)=[X, Y]_{h}-\left[X_{h}, Y_{h}\right]
$$

where $X_{h}: F \rightarrow T F$ is the horizontal lift of a vector field $X: M \rightarrow T M$. According to the definition of the horizontal space $H_{u}$ given above, the horizontal lift of a vector field $X$ is given, for $u \in F_{x}$, by

$$
X_{h}(u)=\sigma_{\frac{u}{2}} \sigma_{0_{x}}(X(x))
$$

By homogenity, it suffices to calculate $\Omega_{o}$, the value at the base point, and then it is enough to plug in the vector fields $X=\tilde{v}, Y=\tilde{w} \in \mathfrak{m}$ having value $X(o)=v, Y=w$ for $v, w \in T_{o} M$. This already implies that $[X, Y]_{o}=0$, and so we are left with calculating $\left[\tilde{v}_{h}, \tilde{w}_{h}\right]_{u}$. This can be done by a calculation in a chart, corresponding essentially to [5, Lemma 26.4]; the outcome is $[v, w, u]$, as expected.

(b) Approaches using higher-order tangent bundles. We analyze the structure of the bundles $T F$ and $T T F$ in exactly the same way as done in [5, Chapter 27], for the case of the tangent bundle: as mentioned in the proof of the preceding theorem, $T F$ has abelian fibers defining the canonical connection, and TTF is non-abelian, leading to an intrinsic description of the curvature in terms of the symmetric space structure of the fibers. The conclusion is the same as with $T^{3} M: \Omega(u, v) w=[u, v, w]$ for $u, v, w$ belonging to the three "axes" $\varepsilon_{1} T_{p} M, \varepsilon_{2} T_{p} M, F_{p}$ of TTF.

\subsection{Extension problem: on existence}

Via Theorem 5.3, we can relate the extension problem to a problem on holonomy representations: assume $M=G / H$ is a homogeneous symmetric space and assume given a representation $\rho$ : $H \rightarrow \mathrm{GL}(V)$; then the associated bundle $F=G \times_{H} V$ carries a tensor field of curvature type $r: \mathfrak{m} \wedge \mathfrak{m} \rightarrow \mathfrak{h} \rightarrow \mathfrak{g l}(V)$, coming from the derived representation $\dot{\rho}: \mathfrak{h} \rightarrow \mathfrak{g l}(V)$. Can we find a connection (coming from a symmetric bundle structure on $F$ ) such that $r$ is its curvature, i.e., such that this representation becomes a holonomy representation? In case of the tangent bundle, $F=T M$, the answer clearly is positive, since $H$ is the holonomy group of the canonical connection on the tangent bundle.

\section{Symmetric structures on the tangent bundle}

In this chapter we will show that, for a rather big class of symmetric spaces $M$, the tangent bundle $T M$ carries (at least) two different symmetric structures with the same underlying reflection space structure. For instance, this is the case for the general linear group, seen as symmetric space. 


\subsection{Example: the general linear group}

The tangent bundle of the group $\operatorname{GL}(n, \mathbb{K})$ can be identified with the general linear group over the dual numbers $\mathbb{K}[\varepsilon]$,

$$
T \mathrm{GL}(n, \mathbb{K})=\mathrm{GL}(n, \mathbb{K}[\varepsilon])=\{g+\varepsilon X \mid g \in \mathrm{GL}(n, \mathbb{K}), X \in M(n, \mathbb{K})\}
$$

with $\varepsilon$-bilinear multiplication $(g+\varepsilon X)(h+\varepsilon Y)=g h+\varepsilon(X h+g Y)$. The canonical symmetric space structures on $\mathrm{GL}(n, \mathbb{K})$ and on its tangent bundle are given by the product map $\mu(g, h)=g h^{-1} g$ (see Example 2.1).

Theorem 7.1. The vector bundle $T \mathrm{GL}(n, \mathbb{K})$ admits a second symmetric bundle structure isomorphic to the homogeneous symmetric space

$$
L / K=\mathrm{GL}(n, \mathbb{D}) / \mathrm{GL}(n, \mathbb{K}[\varepsilon])
$$

where $\mathbb{D}$ is the noncommutative ring of "degenerate quaternions over $\mathbb{K}$ ",

$$
\mathbb{D}=\left\{\left(\begin{array}{cc}
a & b \\
\bar{b} & \bar{a}
\end{array}\right) \mid a, b \in \mathbb{K}[\varepsilon]\right\}
$$

with group involution of $L$ induced by conjugation of $\mathbb{D}$ with respect to its subalgebra of diagonal matrices.

Proof. Before coming to details of the calculation, let us give a heuristic argument: the "Hermitian complexification" of the group $\operatorname{GL}(n, \mathbb{R})$ is the symmetric space $M_{h \mathbb{C}}=\mathrm{GL}(2 n, \mathbb{R}) / \mathrm{GL}(n, \mathbb{C})$ of complex structures on $\mathbb{R}^{2 n}$ (see [3, Chapter IV]). In principle, in the present context we should replace complex structures $\left(I^{2}=-\right.$ id) by "infinitesimal structures" $\left(E^{2}=0\right)$, but this attempt fails since $E$ is not invertible. However, changing the point of view by considering a complex structure on $\mathbb{R}^{2 n}$ rather as a " $\mathbb{C}^{n}$-form of the algebra $(M(2,2 ; \mathbb{R}))^{n}$ ", and viewing $\mathrm{GL}(2 n, \mathbb{R})$ rather as $\operatorname{GL}(n, M(2,2 ; \mathbb{R}))$, the suitably reformulated arguments carry over from the CayleyDickson extension $\mathbb{C} \subset M(2,2 ; \mathbb{R})$ to the "degenerate Cayley-Dickson extension" $\mathbb{R}[\varepsilon] \subset \mathbb{D}$ (and in fact to any extension à la Cayley-Dickson of a commutative ring with nontrivial involution, see below). In the following calculations we need the matrices

$$
F_{n}=\left(\begin{array}{cc}
0 & \mathbf{1}_{n} \\
\mathbf{1}_{n} & 0
\end{array}\right), \quad I_{n, n}=\left(\begin{array}{cc}
\mathbf{1}_{n} & 0 \\
0 & -\mathbf{1}_{n}
\end{array}\right), \quad R_{n}:=\left(\begin{array}{cc}
\mathbf{1}_{n} & \mathbf{1}_{n} \\
-\mathbf{1}_{n} & \mathbf{1}_{n}
\end{array}\right)
$$

Let us abbreviate $\mathbb{A}:=\mathbb{K}[\varepsilon]$; then

$$
\mathbb{D}=\left\{X \in M(2,2 ; \mathbb{A}) \mid F_{1} X F_{1}=\bar{X}\right\}
$$

where $\overline{A+\varepsilon B}=A-\varepsilon B$. We call the map $\tau: \mathbb{D} \rightarrow \mathbb{D}, X \mapsto I_{1,1} X I_{1,1}$ an $\mathbb{A}$-form of $\mathbb{D}$; this is justified by the fact that the fixed ring $\mathbb{D}^{\tau}$ is the ring of diagonal matrices in $\mathbb{D}$, which is isomorphic to $\mathbb{A}$, and that $\tau$ anticommutes with the "structure map" $f: \mathbb{D} \rightarrow \mathbb{D}, X \mapsto F X$. For the corresponding $\mathbb{K}$-linear maps $\mathbb{D}^{n} \rightarrow \mathbb{D}^{n}$ we will again write $\tau$ and $f$ instead of $\tau^{n}$ and $f^{n}$. 
The group

$$
\begin{aligned}
\operatorname{GL}(n, \mathbb{D}) & =\left\{g \in \operatorname{GL}(2 n, \mathbb{A}) \mid F_{n} g F_{n}=\bar{g}\right\} \\
& =\left\{g=\left(\begin{array}{cc}
a & b \\
\bar{b} & \bar{a}
\end{array}\right) \mid a, b \in M(n, n ; \mathbb{A}), \operatorname{det}(g) \in \mathbb{K}^{\times}\right\}
\end{aligned}
$$

acts on the space $\operatorname{End}_{\mathbb{K}}\left(\mathbb{D}^{n}\right)$ by conjugation. The stabilizer of $\tau$ is given by $b=0$, i.e., it is the group $\operatorname{GL}(n, \mathbb{A})$. Thus the $\mathrm{GL}(n, \mathbb{D})$-orbit of $\tau$ is a homogeneous symmetric space:

$$
\mathcal{O}:=\mathrm{GL}(n, \mathbb{D}) \cdot \tau \cong \mathrm{GL}(n, \mathbb{D}) / \mathrm{GL}(n, \mathbb{A})
$$

We will show now that $\mathcal{O}$ is a vector bundle over $\operatorname{GL}(n, \mathbb{K})$, and that this vector bundle is isomorphic to the homogeneous bundle $\operatorname{GL}(n, \mathbb{A})$ over $\operatorname{GL}(n, \mathbb{K})$. To this end, observe that the group $\mathrm{GL}(n, \mathbb{D})$ contains a subgroup isomorphic to $\mathrm{GL}(n, \mathbb{K}) \times \mathrm{GL}(n, \mathbb{K})$, namely the group of matrices of the form

$$
\left(\begin{array}{ll}
g+h & h-g \\
h-g & g+h
\end{array}\right)=R_{n}\left(\begin{array}{ll}
g & 0 \\
0 & h
\end{array}\right) R_{n}^{-1}
$$

with $g, h \in \mathrm{GL}(n, \mathbb{K})$. Now let this subgroup act on $\tau$ (whose matrix is $I_{n, n}$ ):

$$
R_{n}\left(\begin{array}{ll}
g & 0 \\
0 & h
\end{array}\right) R_{n}^{-1} I_{n, n} R_{n}\left(\begin{array}{cc}
g^{-1} & 0 \\
0 & h^{-1}
\end{array}\right) R_{n}^{-1}=R_{n}\left(\begin{array}{cc}
0 & g h^{-1} \\
h g^{-1} & 0
\end{array}\right) R_{n}^{-1}
$$

The stabilizer of $\tau$ is gotten by taking $g=h$, and so the orbit of $\tau$ under this group is isomorphic to the symmetric space $\mathrm{GL}(n, \mathbb{K}) \times \mathrm{GL}(n, \mathbb{K}) /$ diag $\cong \mathrm{GL}(n, \mathbb{K})$ (group case). On the other hand, $\mathrm{GL}(n, \mathbb{D})$ contains the abelian normal subgroup of matrices of the form

$$
\mathbf{1}_{2 n}+\varepsilon\left(\begin{array}{cc}
X & Y \\
-Y & -X
\end{array}\right)
$$

with $X, Y \in M(n, n ; \mathbb{A})$, and $\mathrm{GL}(n \mathbb{D})$ is a semidirect product of these two subgroups. It follows that $\mathcal{O}$ is a vector bundle over the orbit $\mathrm{GL}(n, \mathbb{K}) \times \mathrm{GL}(n, \mathbb{K}) / \operatorname{diag} \cong \mathrm{GL}(n, \mathbb{K})$. Let us determine the fiber over the base point $F_{n}$. Since

$$
(\mathbf{1}+\varepsilon Z) F_{n}(\mathbf{1}-\varepsilon Z)=F_{n}+\varepsilon\left(Z F_{n}-F_{n} Z\right)
$$

the stabilizer is gotten by $X=0$, whereas for $Y=0$ we get the fiber of $\mathcal{O}$ over the base point:

$$
\left(\begin{array}{cc}
\mathbf{1} & \varepsilon X \\
-\varepsilon X & \mathbf{1}
\end{array}\right), \quad X \in M(n, n ; \mathbb{K})
$$

Thus the fiber is isomorphic to $M(n, n ; \mathbb{K})$, and hence $\mathcal{O}$ is isomorphic as a homogeneous bundle to $\operatorname{GL}(n, \mathbb{A})$. The remaining task of calculating the Lts of the symmetric space $\mathcal{O}$ becomes easier by transforming everything with the "Cayley transform" $R_{n}$ : the Cayley transformed version of $\mathfrak{g l}(n, \mathbb{D})$ is

$$
R_{n} M(n, n ; \mathbb{D}) R_{n}^{-1}=\left\{\left(\begin{array}{cc}
a & \varepsilon b \\
\varepsilon c & d
\end{array}\right) \mid a, b, c, d \in M(n, n ; \mathbb{K})\right\}
$$


The Lie algebra of $\operatorname{GL}(n, \mathbb{A})$ is imbedded as the subalgebra given by the conditions $a=d$ and $b=c$. The tangent spaces of our two special orbits inside $R_{n} \mathcal{O} R_{n}^{-1}$ are complementary to this subalgebra, namely

$$
\mathfrak{m}=\left\{\left(\begin{array}{cc}
Y & 0 \\
0 & -Y
\end{array}\right) \mid Y \in M(n, n ; \mathbb{K})\right\}, \quad V=\left\{\left(\begin{array}{cc}
0 & \varepsilon X \\
-\varepsilon X & 0
\end{array}\right) \mid X \in M(n, n ; \mathbb{K})\right\}
$$

so that $\mathfrak{m} \oplus V$ is the Lts of $\mathcal{O}$, where the triple product is the usual triple Lie bracket of matrices since the group action is by ordinary conjugation of matrices. The formula shows that $\mathfrak{m}$ clearly is isomorphic to the usual $\operatorname{Lts}$ of $\operatorname{GL}(n, \mathbb{K})$, that $V$ is abelian and that $\operatorname{GL}(n, \mathbb{K})$ acts on $V$ by conjugation, i.e., the $r$-component of the corresponding Lts representation of $\mathfrak{m}$ is the usual one (corresponding to the fact that $\mathcal{O}$ is isomorphic to $\mathrm{GL}(n, \mathbb{K}[\varepsilon]$ ) as a homogeneous bundle). However, the whole Lts representation just defined is not equivalent to the adjoint Lts representation of $\mathfrak{g l}(n, \mathbb{K})$ on $\varepsilon \mathfrak{g l}(n, \mathbb{K})$. In fact, the corresponding involutive Lie algebras $(\mathfrak{g l}(n, \mathbb{D}), \mathfrak{g l}(n, \mathbb{A}))$ and $(\mathfrak{g l}(n, \mathbb{A}) \times \mathfrak{g l}(n, \mathbb{A})$, dia $)$ are not isomorphic: already for $n=1$, they are not isomorphic since $\mathfrak{g l}(1, \mathbb{A}) \times \mathfrak{g l}(1, \mathbb{A})=\mathbb{A} \times \mathbb{A}$ is commutative, whereas $\mathfrak{g l}(1, \mathbb{D})=\mathbb{D}$ is not.

As mentioned above, instead of dual numbers we could have taken for $\mathbb{A}$ another ring extension of the form $\mathbb{A}_{\mu}=\mathbb{K}[X] /\left(X^{2}-\mu\right)$ with arbitrary $\mu \in \mathbb{K}$ instead of $\mu=0$. Then the symmetric space $\mathrm{GL}(n, \mathbb{K})$ has two different scalar extensions from $\mathbb{K}$ to $\mathbb{A}_{\mu}$ : the "straight one", simply gotten by taking the group case $\operatorname{GL}\left(n, \mathbb{A}_{\mu}\right)$, and another, "twisted" one, given by the homogeneous symmetric space $\operatorname{GL}\left(n, \mathbb{D}_{\mu}\right) / \mathrm{GL}\left(n, \mathbb{A}_{\mu}\right)$, where $\mathbb{D}_{\mu}$ is the split Cayley-Dickson extension of $\mathbb{A}_{\mu}$ (see [6] for details). One could even replace here the algebra of square matrices by any other associative $\mathbb{K}$-algebra. For $\mu=-1$, we are back in the example of the "twisted complexification of $\operatorname{GL}(n, \mathbb{R})$ ". It is interesting that the interpretation of $\mathcal{O}$ as the "space of complex structures" (i.e., endomorphisms with $E^{2}=\mu$ ) works only for invertible scalars $\mu$, whereas the interpretation given here works uniformly for all scalars.

\subsection{Jordan-extensions}

Besides general linear groups, for all other "classical" symmetric spaces, there exist similar descriptions of symmetric bundle structures on the tangent bundle, see [6] for the case of Grassmannians, Lagrangians and orthogonal groups. The latter example gives rise to the " $\mathbb{D}$-unitary groups", analogs of $\operatorname{Sp}(n)$ with $\mathbb{H}$ replaced by $\mathbb{D}$. The general construction principle behind these examples uses Jordan theory:

Definition 7.2. A Jordan-extension of an Lts $(\mathfrak{m}, R)$ is given by a Jordan triple product $T$ : $\mathfrak{m}^{3} \rightarrow \mathfrak{m}$ such that

$$
R(X, Y) Z=T(X, Y, Z)-T(Y, X, Z)
$$

Recall that a Jordan triple system $(\mathrm{Jts})$ is a linear space $\mathfrak{m}$ together with a trilinear map $T: \mathfrak{m}^{3} \rightarrow \mathfrak{m}$ such that

(JT1) $T$ is symmetric in the outer variables: $T(u, v, w)=T(w, v, u)$

(JT2) $T(u, v, T(x, y, z))=T(T(u, v, x), y, z)-T(x, T(v, u, y), z)+T(x, y, T(u, v, z))$

For any Jts $T$, the trilinear map defined by $R_{T}(x, y) z=T(x, y, z)-T(y, x, z)$ is an Lts: we call the correspondence $T \mapsto R_{T}$ the Jordan-Lie functor (cf. [3]). 
Theorem 7.3 (the twisted regular representation defined by a Jordan extension). Assume $(\mathfrak{m}, R)$ is an Lts having a Jordan extension $T$. Let $(\varepsilon \mathfrak{m}, r, m)$ be the regular representation of $\mathfrak{m}$ (recall that the corresponding split null extension $\mathfrak{m} \oplus \varepsilon \mathfrak{m}$ is just the scalar extension by dual numbers). Then there exists another representation $(\varepsilon \mathfrak{m}, \tilde{r}, \tilde{m})$ of $\mathfrak{m}$, in general, not isomorphic to the regular representation, but having the same $r$-component (i.e., $\tilde{r}=r$ ).

Proof. We follow the lines of the proof of the corresponding statement for complexifications in [3, Chapter III]: let $\mathfrak{m}[\varepsilon]=\mathfrak{m} \oplus \varepsilon \mathfrak{m}$ and $T[\varepsilon]: \mathfrak{m}_{\varepsilon}^{3} \rightarrow \mathfrak{m}_{\varepsilon}$ be the $\epsilon$-trilinear scalar extension of $T$ by dual numbers. Then the conjugation

$$
\tau: \mathfrak{m}[\varepsilon] \longrightarrow \mathfrak{m}[\varepsilon], \quad \tau(x+\varepsilon y)=\overline{x+\varepsilon y}=x-\varepsilon y
$$

is a $\mathbb{K}$-automorphism of $T[\epsilon]$. But for any involutive $\mathbb{K}$-automorphism, the " $\tau$-isotope"

$$
\tilde{T}(u, v, w):=T[\varepsilon](u, \tau(v), w)
$$

is again a Jts (cf. [3, Lemma III.4.5] for the easy proof). Moreover, this new Jts is $\varepsilon$-linear in the outer variables and $\varepsilon$-antilinear in the inner variable, and since $\tau$ acts trivially on $\mathfrak{m}$, restriction of this new Jts to $\mathfrak{m}^{3}$ gives us back $T$ again. Now we let

$$
\tilde{R}(X, Y):=R_{\tilde{T}}(X, Y)=\tilde{T}(Y, X)-\tilde{T}(X, Y)
$$

This is an Lts which coincides with $R$ on $\mathfrak{m}$ since $T$ was chosen to be a Jordan extension of $R$. Next, $\varepsilon \mathfrak{m}$ is an ideal of $\tilde{R}$ : by (anti-)linearity it is an ideal of $\tilde{T}$, and hence it is one of $\tilde{R}$. Finally, if two terms belong to $\varepsilon \mathfrak{m}$, then application of $\tilde{T}$ gives zero, and therefore also application of $\tilde{R}$ gives zero. Thus $\tilde{\mathfrak{m}}$ is an Lts having the properties from Lemma 3.2, and hence is the split null extension corresponding to a representation $(\tilde{r}, \tilde{m})$ on $\varepsilon \mathfrak{m}$.

Now we show that $r=\tilde{r}$ : for $x, y, v \in \mathfrak{m}$,

$$
\tilde{r}(x, y, \varepsilon v)=\tilde{R}(x, y, \varepsilon v)=\tilde{T}(x, y, \varepsilon v)-\tilde{T}(y, x, \varepsilon v)=r(x, y, \varepsilon v)
$$

In order to prove that $(r, m)$ and $(\tilde{r}, \tilde{m})$ are in general not isomorphic, observe that the split null extension of $(r, m)$, being just scalar extension by $\mathbb{K}[\varepsilon]$, has the property that $R(\varepsilon X, Y)=$ $R(X, \varepsilon Y)$. On the other hand,

$$
\tilde{R}(\varepsilon X, Y)=\tilde{T}(\varepsilon X, Y, \cdot)-\tilde{T}(Y, \varepsilon X, \cdot)=\varepsilon(\tilde{T}(X, Y)+\tilde{T}(Y, X))=-\tilde{R}(X, \varepsilon Y)
$$

Thus $R$ and $\tilde{R}$ cannot belong to isomorphic representations unless they vanish both. (This does not exclude that, as Lie triple systems over $\mathbb{K}$, they may be isomorphic in special cases.)

\subsection{Final comments}

Essentially, all classical Lie triple systems (and about half of the exceptional ones) admit Jordan extensions (cf. [3, Chapter XII]). For instance, it is easily checked that $M(n, n ; \mathbb{K})$ with the triple product $T(u, v, w)=u v w+w v u$ is a $\mathrm{Jts}$, and then

$$
T(u, v, w)-T(v, u, w)=u v w+w v u-(v u w+w u v)=[u, v] w-w[u, v]=[[u, v], w]
$$

so that we have a Jordan extension of $\mathfrak{g l}(n, \mathbb{K})$. Correspondingly, $\operatorname{GL}(n, \mathbb{K})$ and essentially all classical symmetric spaces admit on their tangent bundle a symmetric bundle structure that is different from the usual one. We conjecture that (at least for simple finite-dimensional Lts over $\mathbb{R}$ or $\mathbb{C}$ ) all symmetric bundle structures on the tangent bundle are exactly of two types: 
(1) "straight": given by the canonical symmetric structure on $T M$, corresponding to the regular representation of the Lts $\mathfrak{m}$,

(2) "twisted": given by the construction from the preceding theorem.

This conjecture is of course supported by the corresponding fact for complexifications of symmetric spaces, which (for simple finite-dimensional Lts over $\mathbb{R}$ ) are either straight or twisted (see [3, Corollary V.1.12]). However, the proof given in loc. cit. for the complex and para-complex cases does not carry over to the tangent case (the invertibility of $i$, resp. $j$, in $\mathbb{K}[i]$, resp. $\mathbb{K}[j]$, is used at a crucial point, and $\varepsilon$ clearly is not invertible in $\mathbb{K}[\varepsilon]$ ). A proof covering all three cases at the same time would be of great value for a better understanding of the "Jordan-Lie functor" (see [3]), and it should relate the "extension problem for the Jordan-Lie functor" with the extension problem for Lts representations as discussed here. One might conjecture that an interpretation in terms of the Cayley-Dickson process, which turned out to be useful in the special case of $\mathrm{GL}(n, \mathbb{K})$, could be the key for proving the conjecture, but this is not clear at present.

\section{Acknowledgement}

The first author would like to thank the Hausdorff Institute (Bonn) for hospitality when part of this work was carried out.

\section{References}

[1] M. Barr. Cartan-Eilenberg cohomology and triples. J. Pure Applied Algebra, 112 (1996), 219-238.

[2] J. M. Beck. Triples, Algebras and Cohomology. Ph.D. Thesis, Columbia University, 1967, http://www.emis.de/journals/TAC/reprints.

[3] W. Bertram. The Geometry of Jordan and Lie Structures. Lecture Notes in Math., 1754, Springer, Berlin, 2000.

[4] W. Bertram. Generalized projective geometries: General theory and equivalence with Jordan structures. Adv. Geom., 2 (2002), 329-369.

[5] W. Bertram. Differential geometry, Lie groups and symmetric spaces over general base fields and rings. Mem. Amer. Math. Soc., 192 (2008), x+202.

[6] M. Didry. Structures algébriques associées aux espaces symétriques. Thesis, Institut Elie Cartan, Nancy, 2006.

[7] M. Didry. Construction of groups associated to Lie- and Leibniz algebras. J. Lie theory, 17 (2007), 399-426.

[8] S. Eilenberg. Extensions of general algebras. Ann. Soc. Pol. Math., 21 (1948), 125-134.

[9] J. Faraut and A. Korànyi. Analysis on Symmetric Cones. Clarendon Press, Oxford, 1994.

[10] B. Harris. Cohomology of Lie triple systems and Lie algebras with involution. Trans. Amer. Math. Soc., 98 (1961), 148-162.

[11] T. L. Hodge and B. J. Parshall. On the representation theory of Lie triple systems. Trans. Amer. Math. Soc., 354 (2002), 4359-4391.

[12] N. Jacobson. General representation theory of Jordan algebras. Trans. Amer. Math Soc., 70 (1951), 509-530.

[13] S. Kobayashi and K. Nomizu. Foundations of Differential Geometry. Volume II. Wiley, New York, 1969.

[14] W. G. Lister. A structure theory for Lie triple systems. Trans. Amer. Math. Soc., 72 (1952), 217-242.

[15] O. Loos. Spiegelungsräume und homogene symmetrische Räume. Math. Z., 99 (1967), 141-170. 
[16] O. Loos. Symmetric Spaces I. Benjamin, New York, 1969.

[17] O. Loos. Representations of Jordan triples. Trans. Amer. Math. Soc., 185 (1973), 199-211.

[18] O. Loos. Jordan Pairs. Lecture Notes in Math., 460, Springer, Berlin, 1975.

[19] J. Mostovoy and J. M. Pérez-Izquierdo. Ideals in non-associative universal enveloping algebras of Lie triple systems. Preprint arXiv:math/0506179, 2005.

[20] L. V. Sabinin. Smooth quasigroups and loops. Mathematics and its Applications, 492, Kluwer Academic Publishers, Dordrecht, 1999.

[21] O. Smirnov. Imbedding of Lie triple systems into Lie algebras. Preprint arXiv:math/0906.1170, 2009.

Received January 9, 2009

Revised May 07, 2009 